\title{
Recovery Slope of Heart Rate Variability as an Indicator of Internal Training Load
}

\author{
José Naranjo Orellana ${ }^{1 *}$, Claudio Nieto-Jiménez ${ }^{2}$, José F. Ruso-Álvarez ${ }^{1}$ \\ ${ }^{1}$ Pablo de Olavide University, Sevilla, Spain \\ ${ }^{2}$ Universidad del Desarrollo, Santiago, Chile \\ mail: jinarore@upo.es
}

How to cite this paper: Naranjo Orellana, J., Nieto-Jiménez, C. and Ruso-Álvarez, J.F. (2019) Recovery Slope of Heart Rate Variability as an Indicator of Internal Training Load. Health, 11, 211-221.

https://doi.org/10.4236/health.2019.112019

Received: January 24, 2019

Accepted: February 18, 2019

Published: February 21, 2019

Copyright (c) 2019 by author(s) and Scientific Research Publishing Inc. This work is licensed under the Creative Commons Attribution International License (CC BY 4.0).

http://creativecommons.org/licenses/by/4.0/

(c) (i) Open Access

\begin{abstract}
The way in which the Root Mean Square of the Successive Differences between adjacent RR intervals (RMSSD) recovers immediately after exercise could be a good indicator of internal training load (ITL). The aim of this study is to design a recovery index based on RMSSD. Forteen healthy men took part in this study. The experiment lasted 2 weeks, with 4 separate (48 $72 \mathrm{~h}$ ) sessions. First session was an incremental treadmill test to determine ventilatory thresholds (VT1 and VT2) and maximal aerobic speed (MAS). Each subject ran at VT1 speed (second day), VT2 speed (third day) and a time-to-exhaustion test at MAS (fourth day). The duration of VT1 and VT2 loads was selected in such a way that the product intensity-duration (training load) was the same. HRV was measured from 10' prior to test (Rest) to $30^{\prime}$ after completed (Recovery). Recovery slopes were calculated from RMSSD values at 10 and 30 minutes. Borg scale was recorded at the end of every test and the Training Impulse (TRIMP) values were calculated using Banister equations. The RMSSD values dropped substantially regardless of the intensity and the duration of exercise (average $4 \mathrm{~ms}$ ). The RMSSD recovery was linear during the $30 \mathrm{~min}$ and different depending on the intensity of exercise. To propose a recovery index, we calculated the slope of RMSDD over the $30 \mathrm{mi}-$ nutes (slope-30) and also the first 10 minutes (slope-10). Given that the slopes presented an exponential behavior in relation with effort intensity, three curves were obtained (average values, plus SD and minus SD) defining a nomogram. For practical application, we propose: 1 ) to measure RMSSD the last 5 minutes of exercise and any period of 5 minutes during the first 30 minutes recovery; 2) to calculate the slope of RMSSD between exercise and recovery; 3) to compare with the nomogram.
\end{abstract}

\section{Keywords}

Training Load, Recovery, Hrv, Rmssd, Parasympathetic 


\section{Introduction}

There is a great deal of interest in controlling training load (TL) in athletes [1], distinguishing between the load administered or external training load (ETL) and the way in which each subject assimilates and responds to the administrated load, which is known as internal training load (ITL) [2]. Given that heart rate variability (HRV) [3] is a non-invasive tool capable of evaluating sympathetic and parasympathetic modulation [4] [5], it has been proposed as a valid method to evaluate individual response to a given work load and, therefore, training load [6] [7]. To evaluate the specific response of the sympathetic-parasympathetic equilibrium to a specific work load, it is reasonable to think that HRV will fundamentally evaluate ITL, particularly if the object of analysis is the reactivation of the parasympathetic system after exercise [8].

However, there are still discrepancies and some conflicting results that prevent clear conclusions from being reached that could be applied simply and directly to the control of training load. In fact, the most recent meta-analysis that analyzes the relationship between measures of ITL and ETL [2] does not include any measure of HRV, and almost all studies that have examined ITL use a rating of perceived exertion of the training session (sRPE and its different variants) [9] or Training Impulse (TRIMP) [10].

Most HRV studies evaluate the changes in different variables during recovery, immediately after exercise to evaluate how these values recover [11]. There is widespread agreement that, changes in HRV at low intensity exercise are related with intensity but not with duration [11] [12] [13] [14], so that the immediate recovery of parasympathetic variables is conditioned principally by exercise intensity [5]. However, other authors report that exercise duration must be considered when investigating post-exercise HRV, even at low intensity [11].

Furthermore, the prevailing idea in the literature is that the most useful approach is to explore different exercise intensities [12] [15] [16] and the time domain variables offer fewer discrepancies than frequency domain variables. In the time domain, RMSSD (Root Mean Square of the Successive Differences between adjacent RR intervals in $\mathrm{ms}$ ) is the most used variable in sport due to the lower coefficient of variation compared with other indices [17]. In addition, it is known that RMSSD is lower than $5 \mathrm{~ms}$ at moderate intensity exercise [11].

To our knowledge, most of previous studies did not adjust intensity and volume to obtain the same TL. Furthermore, no useful HRV post-exercise indexes can be easily applied for the control of TL, although two indexes have been previously reported [8] [18].

Our hypothesis is that the way in which RMSSD recovers immediately after exercise could be a good indicator of ITL. So, the aim of this study is to design a recovery index based on RMSSD and to propose the way to use it in daily training.

\section{Methods}

\subsection{Subjects}

A total of 14 men (Sports Science students) took part in this study during 2 
weeks, all of whom were healthy, non-smokers and physically active but not participating in any athletic sport (age $20.93 \pm 1.38$ years; weight $75.34 \pm 10.07 \mathrm{~kg}$; height $\left.178.04 \pm 5.83 \mathrm{~cm} ; \mathrm{VO}_{2 \max } 49.33 \pm 3.93 \mathrm{ml} \cdot \mathrm{kg}^{-1} \cdot \mathrm{min}^{-1}\right)$. It was a convenience sample and highly homogeneous.

Following the general indications of the Task Force [3], all the subjects were asked not to drink alcohol or caffeinated beverages, and to abstain from physical activity during the 24 hours prior to each test.

Each subject was given a medical questionnaire to ensure that they were not receiving treatments or suffering from cardiovascular or any other disorder that might alter the state of the autonomic nervous system. All the subjects were informed of the procedure that would be followed, and they gave their written consent to take part in the experiment. The Local Ethics Board approved the study, which followed all the principles expressed in the Declaration of Helsinki [19].

Experimental design

The experiment lasted a total of 2 weeks, with 4 separate sessions with 48 - 72 $\mathrm{h}$ intervals between each one, taking place at approximately the same time of day $(10: 00 \pm 2 \mathrm{~h})$ and maintaining stable environmental conditions (temperature and humidity).

At the first session, a background questionnaire was completed for each subject, and all the participants' height and weight measurements were taken. An incremental and maximal cardiopulmonary exercise test was performed on an ErgoRun Medical 8 treadmill (Daum Electronic; Fürth, Germany) following a steps protocol with an initial speed of $7 \mathrm{~km} / \mathrm{h}$ for 3 minutes and increases of 1 $\mathrm{km} / \mathrm{h}$ each minute until volitional exhaustion. A slope of $1 \%$ was fixed during the entire test. The test was carried out using a Breezesuite CPX ergospirometric equipment (Medical Graphics; St. Paul, Minnesota, USA) calibrated prior to each measurement. The ventilation data were obtained breath by breath using a differential pressure-based flow-meter, as well as the inspiratory and expiratory fractions of $\mathrm{O}_{2}$ and $\mathrm{CO}_{2}$ based on a galvanic cell and infrared analyser respectively.

For the purposes of this study, ventilatory thresholds (VT1 and VT2) were determined following the method proposed by Skinner and McLellan [20], recording the speed corresponding to each threshold. Maximal oxygen uptake $\left(\mathrm{VO}_{2 \max }\right)$ was determined if a plateau in $\mathrm{VO}_{2}$ was reached. If not, the following two criteria were required: 1) respiratory exchange ratio (RER) higher than 1, 15 and heart rate (HR) higher than age-predicted maximum value (220-age) [21].

Maximal Aerobic Speed (MAS) was assessed as the speed at which $\mathrm{VO}_{2}$ plateau started.

At each session, training load was calculated as the product of intensity (speed) by volume (time) [17]. By expressing speed in $\mathrm{km} / \mathrm{h}$ and time in hours, $\mathrm{TL}$ is expressed as the distance covered in kilometres.

At the second session, each subject ran constantly for 20 minutes at VT1 speed. Given the low intensity, no prior warm-up was needed. 
At the third session, each subject ran constantly at VT2 speed. In order to ensure the same TL, the time was established in such a way that the product (intensity per duration) was the same as for VT1. In this way, the same distance was covered both in VT1 and VT2 test. This test was preceded by a 5 minutes warm-up at $60 \%$ of MAS.

At the fourth session, a time-to-exhaustion test was carried out for Maximal Aerobic Speed (MAS). Given that this speed was very high, it was achieved gradually over a 2 minutes period: 90 seconds at $70 \%$ of MAS followed by 30 seconds at $80 \%$ of MAS.

\subsection{HRV Measures}

At sessions 2, 3 and 4, a V800 Polar HR monitor was fitted onto the subjects, with an H10 Sensor thoracic band (Polar Inc., Kempele, Finland). HRV measurements were recorded from 8 minutes prior to the test until 30 minutes after the test was completed. All the pre and post exercise measurements were taken with the subject in a seated position and in a calm and quiet environment. The subject had to sit down immediately after finishing the test (with no active cool down) in order to measure their recovery.

The RR time series were downloaded using the Polar Flow Sync application (version 2.6.2) to be analyzed using Kubios HRV software (Version 2.1, University of Eastern Finland, Kuopio, Finland).

At each session, the last 5 minutes of the rest and exercise records were taken. In the case of the 30-minute recovery period ( $\mathrm{rec}$ ), measurements were divided into 5-minute slots, starting at minute 10 (rec 10, rec 15, rec 20, rec 25, rec 30). HRV was not quantified the first 5 minute of recovery because of the loss of time series stability derived from the sudden change between the end of the exercise and the start of recovery [22].

Each record was analyzed previously to detect the possible presence of artifacts and anomalous beats, applying the corresponding filters if required.

In order to develop a simple methodology easy to use in real assessments of athletes, analysis was conducted using one variable for parasympathetic stimulus. In accordance with the literature [3], RMSSD was calculated in the time domain as the most widely used statistic when evaluating parasympathetic activity [17] [23].

To propose a recovery index based on HRV, we calculated the recovery slope of the RMSDD values over the course of 30 minutes (slope-30). As in the daily training 30 minutes could be an excessive time, we also tested the 10 minutes RMSSD slope (slope-10).

\subsection{Other Measurements of ITL}

To compare the proposed index, the perceived effort was recorded using the Borg 1 - 10 scale [24] (1 - 2: very light; 3: light; 4 - 5: moderate; 6 - 7: hard; 8 - 9: very hard; 10: extremely hard) and the Training Impulse (TRIMP) values were calculated using Banister equations [10] [25]. 


\subsection{Statistical Analysis}

Firstly, a descriptive study was carried out so that all the data are presented by their average values and standard deviations. The Kolmogorov-Smirnov test was used to verify the normality of the distributions. Then, the Levene Test was applied to verify equality of variances, and an ANOVA was applied using the Games-Howell procedure as the post-hoc test. The level of significance was set at $\mathrm{p}<0.05$, with a confidence level of $95 \%$.

To analyze the relationships between the proposed slopes and other load variables, a Pearson correlation analysis was conducted. Specially, the graphical relationship between RMSSD slopes and intensities was explored.

For statistical analysis, the SPSS programme version 15.0 for Windows was used.

\section{Results}

\subsection{Characteristics of the Test}

Table 1 shows the data for intensity (speed), duration and TL, as well as the Borg scale and TRIMP values for each of the tests. The position of every threshold (VT1 and VT2) is shown as percent of $\mathrm{VO}_{2 \max }$.

\subsection{HRV Data}

Table 2 shows the values for RMSSD measured at rest, in the last 5 minutes of exercise, and over the course of recovery. The values for $\mathrm{p}$ are given, comparing each datum with the value at rest and those for recovery following exercise. There were no significant differences in the baseline values for RMSSD between the tests. RMSSD at all minutes of recovery was significantly different to rest $(\mathrm{P}$ $<0.01)$ at the 3 intensities.

Figure 1 shows that exercise RMSSD is the same at the three intensities explored and its evolution is significantly different from VT1 to the other two, but there was no difference between VT2 and MAS intensities.

\subsection{RMSSD Recovery Slopes}

Table 3 shows the values for Slope-30 and Slope- 10 .

Slope-10 presents a Pearson correlation coefficient ( $r$ ) of 0.09 for TL, -0.70 for Borg's scale, and 0.47 for TRIMP. The $\mathrm{r}$ values for Slope-30 were 0.21 with TL, -0.64 with Borg's scale, and 0.49 with TRIMP.

The Pearson correlation coefficient between Slope-10 and Slope-30 was 0.78.

\section{Discussion}

The main contribution of this study is to provide a simple tool capable of evaluating internal training load based on the immediate recovery of RMSSD following any kind of exertion.

The rest RMSSD values were the same in the different sessions, corresponding to the $75^{\text {th }}$ percentile for the age [26]. 
Table 1. Characteristics of the test.

\begin{tabular}{ccccccc}
\hline & \multicolumn{2}{c}{ VT1 } & \multicolumn{2}{c}{ VT2 } & \multicolumn{2}{c}{ MAS } \\
\cline { 2 - 7 } & Average & SD & Average & SD & Average & SD \\
\hline Speed $(\mathrm{km} / \mathrm{h})$ & 10.24 & 1.44 & 13.71 & 0.89 & 16.29 & 1.1 \\
Time $(\mathrm{h})$ & 0.33 & 0 & 0.22 & 0.05 & 0.11 & 0.03 \\
$\% \mathrm{VO}_{2 \max }$ & 64.39 & 7.22 & 83.11 & 7.32 & 100 & 0 \\
$\mathrm{TL}(\mathrm{km})$ & 3.44 & 0.50 & 3.44 & 0.50 & 1.75 & 0.49 \\
Borg $1-10$ & 3.93 & 0.92 & 7.57 & 1.74 & 9.00 & 0.78 \\
TRIMP & 24.34 & 0.3 & 22.32 & 3.23 & 12.56 & 1.62 \\
\hline
\end{tabular}

Abbreviations: VT1 (first ventilatory threshold); VT2 (second ventilatory threshold); MAS (maximal aerobic speed); $\mathrm{VO}_{2 \max }$ : maximal oxygen uptake; TL (Trainning Load); TRIMP (Trainning Impulse); Note: warm-up ( 5 minutes at $9.77 \pm 0.66 \mathrm{~km} / \mathrm{h}$ ) is not included in the VT2 data, neither the acceleration time (2 minutes at $12.22 \pm 0.82 \mathrm{~km} / \mathrm{h}$ ) in the MAS data.

Table 2. Values of the HRV variables measured at rest, in the last 5 minutes of exercise and throughout the recovery. The $\mathrm{p}$ values compare each situation with the rest and the values of recovery with those of exercise.

\begin{tabular}{|c|c|c|c|c|}
\hline \multicolumn{5}{|c|}{ RMSSD } \\
\hline & & VT1 & VT2 & MAS \\
\hline \multirow[t]{2}{*}{ Rest } & Mean & 71.24 & 71.15 & 68.48 \\
\hline & SD & 31.22 & 21.69 & 19.65 \\
\hline \multirow[t]{3}{*}{ Exc. } & Mean & 3.92 & 4.26 & 4.29 \\
\hline & SD & 1.11 & 0.83 & 0.92 \\
\hline & $\mathrm{p}$ (rest) & 0.000 & 0.000 & 0.000 \\
\hline \multirow[t]{4}{*}{ Rec. 10} & Mean & 19.56 & 7.00 & 6.74 \\
\hline & SD & 10.33 & 3.26 & 3.28 \\
\hline & $\mathrm{p}$ (rest) & 0.001 & 0.000 & 0.000 \\
\hline & $\mathrm{p}(\mathrm{exc})$ & 0.001 & 0.184 & 0.196 \\
\hline \multirow[t]{4}{*}{ Rec. 15} & Mean & 25.39 & 10.07 & 8.11 \\
\hline & $\mathrm{SD}$ & 14.61 & 7.45 & 3.97 \\
\hline & $\mathrm{p}$ (rest) & 0.002 & 0.000 & 0.000 \\
\hline & $\mathrm{p}(\mathrm{exc})$ & 0.002 & 0.149 & 0.051 \\
\hline \multirow[t]{4}{*}{ Rec. 20} & Mean & 27.46 & 11.61 & 8.87 \\
\hline & SD & 14.04 & 6.98 & 4.45 \\
\hline & $\mathrm{p}$ (rest) & 0.003 & 0.000 & 0.000 \\
\hline & $\mathrm{p}(\mathrm{exc})$ & 0.001 & 0.027 & 0.032 \\
\hline \multirow[t]{4}{*}{ Rec. 25} & Mean & 28.59 & 14.00 & 11.28 \\
\hline & $\mathrm{SD}$ & 16.49 & 9.64 & 7.19 \\
\hline & $\mathrm{p}$ (rest) & 0.004 & 0.000 & 0.000 \\
\hline & $\mathrm{p}(\mathrm{exc})$ & 0.002 & 0.035 & 0.045 \\
\hline \multirow[t]{4}{*}{ Rec. 30} & Mean & 33.91 & 16.59 & 11.90 \\
\hline & $\mathrm{SD}$ & 16.45 & 10.43 & 6.16 \\
\hline & $\mathrm{p}$ (rest) & 0.015 & 0.000 & 0.000 \\
\hline & $\mathrm{p}(\mathrm{exc})$ & 0.000 & 0.012 & 0.008 \\
\hline
\end{tabular}

Abbreviations: RMSSD: Root Mean Square of the Successive Differences between adjacent RR intervals in ms.; Exc: exercise; Rec: recovery; VT1 (first ventilatory threshold); VT2 (second ventilatory threshold); MAS (maximal aerobic speed). 
Table 3. Values of recovery slopes of RMSSD during the whole 30 minutes (Slope-30) and in the first 10 minutes (Slope-10).

\begin{tabular}{ccccccc}
\hline & \multicolumn{3}{c}{ Slope-30 } & & \multicolumn{3}{c}{ Slope-10 } \\
\cline { 2 - 7 } & Min. & Avr. & Max. & Min. & Avr. & Max. \\
\hline VT1 & 0.44 & 1.00 & 1.56 & 0.45 & 1.51 & 2.57 \\
VT2 & 0.10 & 0.41 & 0.75 & 0.10 & 0.29 & 0.57 \\
MAS & 0.10 & 0.25 & 0.45 & 0.10 & 0.28 & 0.53 \\
\hline
\end{tabular}

Abbreviations: Min. (minimum); Avr. (average); Max. (maximum); RMSSD: Root Mean Square of the Successive Differences between adjacent RR intervals in ms.; VT1 (first ventilatory threshold); VT2 (second ventilatory threshold); MAS (maximal aerobic speed).

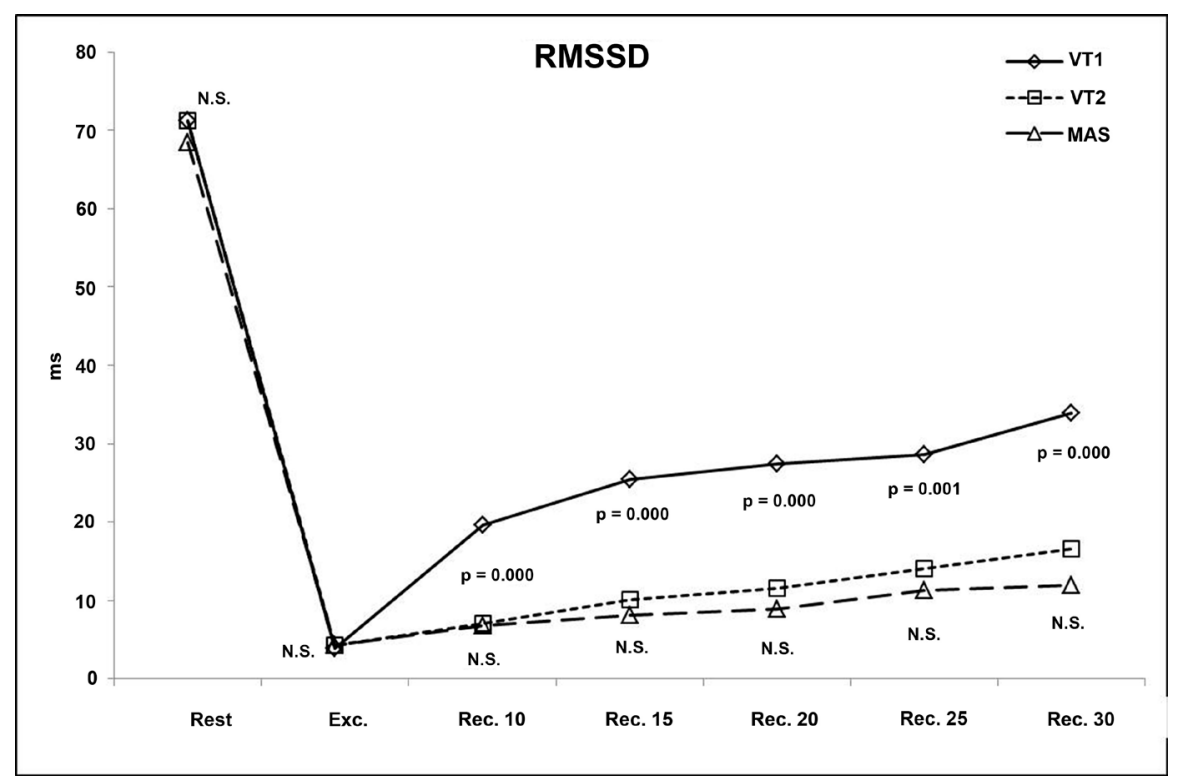

Figure 1. Evolution of RMSSD values in the 3 test. Abbreviations: VT1 (first ventilatory threshold); VT2 (second ventilatory threshold); MAS (maximal aerobic speed); RMSSD: Root Mean Square of the Successive Differences between adjacent RR intervals in ms.; Exc: exercise; Rec: recovery; NS: Non-significant.

The RMSSD reactivation following exercise behaves in a linear way, at least for the first 30 minutes, and the slope can be calculated at any point during those 30 minutes.

The RMSSD values dropped substantially regardless of the intensity and the duration of exercise to an average value of $4 \mathrm{~ms}$ (Figure 1). However, once recovery has begun, we see a progressive increase in the values of the RMSSD, which is significantly faster at the intensity of VT1 than at either of the other two intensities. We agree with other authors [8] [12] who report that the recovery of the RMSSD is progressive and different depending on the intensity of exercise carried out. The main difference between the VT1 tests and the other is intensity, since TL is the same in VT2 and VT1 because the durations of both tests were adjusted according to the intensity. Other studies also find that, at lower intensities, the recovery of the RMSSD is much faster [12] [13]. The recovery of VT2 
and MAS is much slower and with no significant differences, a finding also observed by other authors when carrying out tests at high intensities [15] [27].

Therefore, it would appear that the recovery rate of the parasympathetic system after an exercise load depends mainly on the intensity of the exercise, coinciding with Stanley [5] [11].

The RMSSD recovery slope increases as the intensity of effort decreases, and vice versa. Given that RMSSD recovered faster at lower intensities, if " $n$ " subjects who carry out the same TL undergo the almost total loss of RMSSD regardless of the load carried out [11], but they presented different recovery slopes in their parasympathetic system. We can assume that this recovery slope is an indicator of internal training load (ITL). In other words, the greater the recovery slope, the less the internal load.

We evaluated the slope for all 30 minutes of recovery (Slope-30) and also in the first 10 minutes (Slope-10) looking for an indicator easier to measure in a real situation following training sessions. Slope-10 and Slope-30 had a very good correlation between them and showed very similar correlations with other variables. These slopes have a very low correlation with TL (as expected in a measure of ITL) and when they were compared with other internal load indicators, it was found a moderate correlation with TRIMP, but a very good inverse correlation with Borg scale which is considered one of the best indicators of ITL.

Based on these observations we choose Slope-10 for a practical application given that 30 minutes could be a too long period to ensure the cooperation of athletes.

Table 3 shows the values of Slope-10 that might be expected for each of the intensities explored. Using these data, we generated a nomogram (Figure 2) based on the average and standard deviation values of Slope-10 for each intensity level, given that the VT1 of the subjects was situated at an average intensity of $64 \%$, VT2 at an average intensity of $83 \%$, and MAS was, by definition, $100 \%$. Three exponential curves were obtained, corresponding to the average values, the mean plus SD and the mean minus SD, defining three areas for the recovery slopes for each intensity level. This nomogram can be used as a reference in the assessment of other athletes. The Slope-10 was chosen to construct the nomogram because is an easier period of time to use in real training situations than the full 30 minutes.

Given that the slopes present an exponential behavior in relation with effort intensity, we see that at very high intensities, these exponentials approach asymptote, which is close to 0.1 . For this reason, 0.1 is considered the minimum value of the slopes, so that for any value less than 0.1 , this value would be assigned.

\section{Practical Applications}

The results of this study could be of application for monitoring individual recovery after different workloads (i.e. training sessions). 


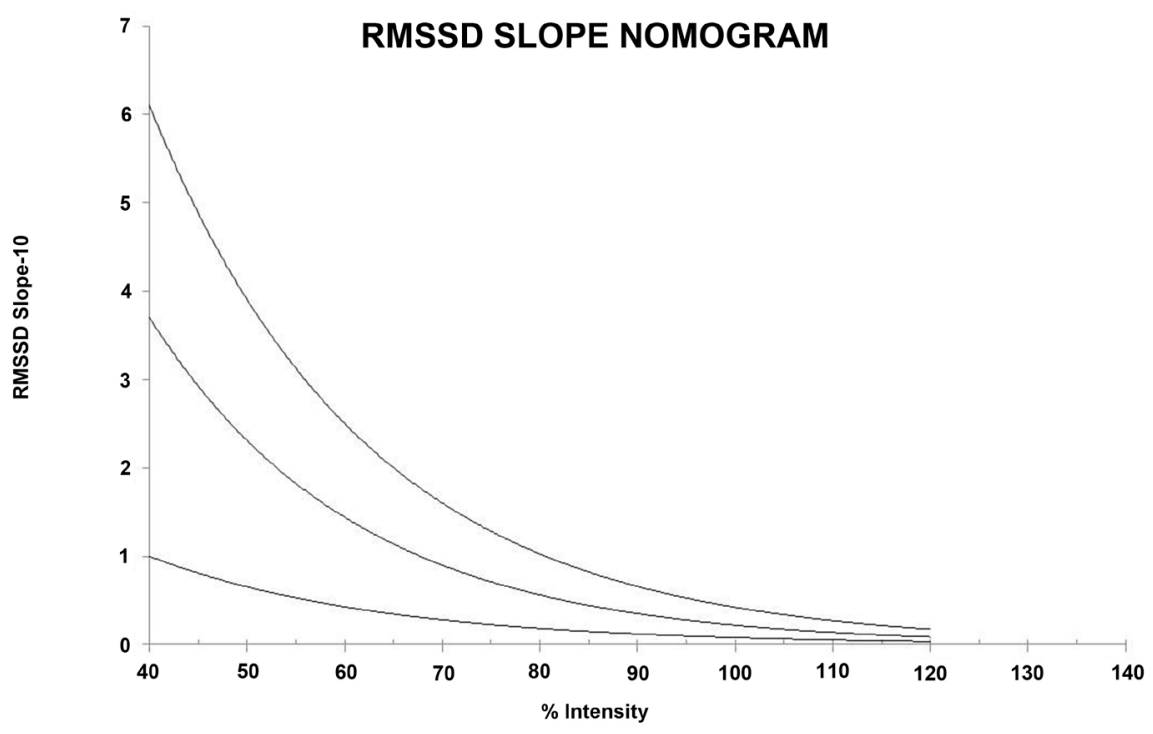

Figure 2. RMSSD slope nomogram. The exponential lines represent the mean values of slope 10 at different intensities (medium line) as well as the medium plus standard deviation (upper line) and the medium minus standard deviation (lower line). (RMSSD: Root Mean Square of the Successive Differences between adjacent RR intervals in ms.)

Given that the RMSSD slope has very similar characteristics along the $30 \mathrm{mi}$ nutes period, we recommend measuring RMSSD along any period of 5 minutes during the first 30 minutes recovery, excluding the first 5 minutes [22]. So, the calculation of the slope would be as follow:

$$
\text { Slope }=(\text { RMSSD-rec. }- \text { RMSSD-exc. }) / t
$$

Were RMSSD-rec is the 5 minutes value during the first 30 minutes recovery, RMSSD-exc. is the value in the last 5 minutes of exercise and $t$ is the time elapsed from the end of exercise to the end of the HRV record. If the RMSSD was not recorded during exercise, we can calculate the RMSSD recovery slope based on the average value of $4 \mathrm{~ms}$.

The main limitation of this study could be the short sample but, although the number of subjects was not very high, it is very similar to the figure included in the majority of studies on HRV. In addition, in this study the same subjects underwent 3 different experimental situations, so the "real" population was 42 subjects.

\section{Conflicts of Interest}

The authors declare no conflicts of interest regarding the publication of this paper.

\section{References}

[1] Bourdon, P.C., Cardinale, M., Murray, A., Gastin, P., Kellmann, M., Varley, M.C., Gabbett, T.J., Coutts, A.J., Burgess, D.J., Gregson, W. and Cable, N.T. (2017) Monitoring Athlete Training Loads : Consensus Statement. International Journal of Physiology and Performance, 12, 161-170. 
[2] McLaren, S.J., Macpherson, T.W., Coutts, A.J., Hurst, C., Spears, I.R. and Weston, M. (2018) The Relationships between Internal and External Measures of Training Load and Intensity in Team Sports: A Meta-Analysis. Sports Medicine, 48, 641-658. https://doi.org/10.1007/s40279-017-0830-Z

[3] Task Force of the European Society of Cardiology and the North American Society of Pacing and Electrophysiology (1996) Heart Rate Variability: Standards of Measurement, Physiological Interpretation and Clinical Use. European Heart Journal, 17, 354-381. https://doi.org/10.1093/oxfordjournals.eurheartj.a014868

[4] Sandercock, G.R.H., Bromley, P.D. and Brodie, D.A. (2005) Effects of Exercise on Heart Rate Variability: Inferences from Meta-Analysis. Medicine and Science in Sports and Exercise, 37, 433-439. https://doi.org/10.1249/01.MSS.0000155388.39002.9D

[5] Stanley, J., Peake, J.M. and Buchheit, M. (2013) Cardiac Parasympathetic Reactivation Following Exercise: Implications for Training Prescription. Sports Medicine, 43, 1259-1277. https://doi.org/10.1007/s40279-013-0083-4

[6] Pichot, V., Roche, F., Gaspoz, J.M., Enjolras, F., Antoniadis, A., Minini, P., Costes, F., Busso, T., Lacour, J.R. and Barthélémy, J.C. (2000) Relation between Heart Rate Variability and Training Load in Middle-Distance Runners. Medicine and Science in Sports and Exercise, 32, 1729-1736. https://doi.org/10.1097/00005768-200010000-00011

[7] Kiviniemi, A.M., Hautala, A.J., Kinnunen, H. and Tulppo, M.P. (2007) Endurance Training Guided Individually by Daily Heart Rate Variability Measurements. European Journal of Applied Physiology, 101, 743-751. https://doi.org/10.1007/s00421-007-0552-2

[8] Goldberger, J.J., Le, F.K., Lahiri, M., Kannankeril, P.J., Ng, J. and Kadish, A.H. (2006) Assessment of Parasympathetic Reactivation after Exercise. American Journal of Physiology-Heart and Circulatory Physiology, 290, H2446-H2452. https://doi.org/10.1152/ajpheart.01118.2005

[9] Oster C. (1998) Monitoring Training in Athletes with Reference to Overtaining Syndrome. Medicine \& Science in Sports \& Exercise, 30, 1164-1168. https://doi.org/10.1097/00005768-199807000-00023

[10] Banister, E.W. and Calvert, T.W. (1980) Planning for Future Performance: Implications for Long Term Training. Canadian Journal of Applied Sport Science, 5, 170-176.

[11] Michael, S., Graham, K.S. and Davis, G.M. (2017) Cardiac Autonomic Responses during Exercise and Post-Exercise Recovery Using Heart Rate Variability and Systolic Time Intervals: A Review. Frontiers in Physiology, 8, 1-19. https://doi.org/10.3389/fphys.2017.00301

[12] Casonatto, J., Tinucci, T., Dourado, A.C. and Polito, M. (2011) Cardiovascular and Autonomic Responses after Exercise Sessions with Different Intensities and Durations. Clinics, 66, 453-458. https://doi.org/10.1590/S1807-59322011000300016

[13] Kaikkonen, P., Nummela, A. and Rusko, H. (2007) Heart Rate Variability Dynamics during Early Recovery after Different Endurance Exercises. European Journal of Applied Physiology, 102, 79-86. https://doi.org/10.1007/s00421-007-0559-8

[14] Seiler, S., Haugen, O. and Kuffel, E. (2007) Autonomic Recovery after Exercise in Trained Athletes: Intensity and Duration Effects. Medicine and Science in Sports and Exercise, 39, 1366-1373. https://doi.org/10.1249/mss.0b013e318060f17d

[15] Kaikkonen, P., Hynynen, E., Mann, T., Rusko, H. and Nummela, A. (2010) Can HRV Be Used to Evaluate Training Load in Constant Load Exercises? European 
Journal of Applied Physiology, 108, 435-442. https://doi.org/10.1007/s00421-009-1240-1

[16] Michael, S., Jay, O., Graham, K.S. and Davis, G.M. (2017) Longer Exercise Duration Delays Post-Exercise Recovery of Cardiac Parasympathetic But Not Sympathetic Indices. European Journal of Applied Physiology, 117, 1897-1906. https://doi.org/10.1007/s00421-017-3673-2

[17] Halson, S.L. (2014) Monitoring Training Load to Understand Fatigue in Athletes. Sports Medicine, 44, 139-147. https://doi.org/10.1007/s40279-014-0253-Z

[18] Saboul, D., Balducci, P., Millet, G., Pialoux, V. and Hautier, C. (2016) A Pilot Study on Quantification of Training Load: The Use of HRV in Training Practice. European Journal of Sport Science, 16, 172-181. https://doi.org/10.1080/17461391.2015.1004373

[19] World Medical Association (2013) WMA Declaration of Helsinki-Ethical Principles for Medical Research Involving Human Subjects.

https://www.wma.net/policies-post/wma-declaration-of-helsinki-ethical-principlesfor-medical-research-involving-human-subjects/

[20] Skinner, J.S. and McLellan, T.H. (1980) The Transition from Aerobic to Anaerobic Metabolism. Research Quarterly for Exercise and Sport, 51, 234-248. https://doi.org/10.1080/02701367.1980.10609285

[21] Howley, E.T., Bassett, J.R. and Welch, H.G. (1995) Criteria for Maximal Oxygen Uptake: Review and Commentary. Medicine \& Science in Sports \& Exercise, 27, 1292-1301. https://doi.org/10.1249/00005768-199509000-00009

[22] Javorka, M., Žila, I., Balhárek, T. and Javorka, K. (2002) Heart Rate Recovery after Exercise: Relations to Heart Rate Variability and Coplexity. Brazilian Journal of Medical and Biological Research, 35, 991-1000. https://doi.org/10.1590/S0100-879X2002000800018

[23] Buchheit, M. and Laursen, P.B. (2007) Parasympathetic Reactivation after Repeated Sprint Exercise. American Journal of Physiology-Heart and Circulatory Physiology, 293, 133-141. https://doi.org/10.1152/ajpheart.00062.2007

[24] Borg, G.A.V. (1982) Phychophysical Bases of Perceived Exertion. Medicine and Science in Sports and Exercise, 14, 377-381. https://doi.org/10.1249/00005768-198205000-00012

[25] Morton, R.H., Fitz-Clarke, J.R. and Banister, E.W. (1990) Modeling Human Performance in Running. Journal of Applied Physiology, 69, 1171-1177. https://doi.org/10.1152/jappl.1990.69.3.1171

[26] Corrales, M.M., de la Cruz Torres, B., Garrido Esquivel, A., Garrido Salazar, M.A. and Naranjo Orellana, J. (2012) Normal Values of Heart Rate Variability at Rest in a Young, Healthy and Active Mexican Population. Health, 4, 377-385. https://doi.org/10.4236/health.2012.47060

[27] de Oliveira, T.P., de Alvarenga Mattos, R., da Silva, R.B.F., Rezende, R.A. and de Lima, J.R.P. (2013) Absence of Parasympathetic Reactivation after Maximal Exercise. Clinical Physiology and Functional Imaging, 33, 143-149. https://doi.org/10.1111/cpf.12009 